Horizons philosophiques

\title{
Le pardon, notion philosophique ou notion religieuse?
}

\section{Jacques Ricot}

Volume 13, numéro 2, printemps 2003

Herder (1744-1803) : le clair-obscur

URI : https://id.erudit.org/iderudit/801241ar

DOI : https://doi.org/10.7202/801241ar

Aller au sommaire du numéro

Éditeur(s)

Collège Édouard-Montpetit

ISSN

1181-9227 (imprimé)

1920-2954 (numérique)

Découvrir la revue

Citer cet article

Ricot, J. (2003). Le pardon, notion philosophique ou notion religieuse? Horizons philosophiques, 13(2), 131-138. https://doi.org/10.7202/801241ar d'utilisation que vous pouvez consulter en ligne.

https://apropos.erudit.org/fr/usagers/politique-dutilisation/ 


\section{LE PARDON, NOTION PHILOSOPHIQUE OU NOTION RELIGIEUSE?}

Peut-on parler du pardon en philosophe ou faut-il abandonner cette notion au registre religieux? Se présente ici un délicat problème de frontière auquel la pensée occidentale a l'habitude de se mesurer et qui engage la philosophie dans la mesure où l'histoire de la philosophie est elle-même une discipline philosophique. Suivant ici une indication de Henri Gouhier dans une étude consacrée à Bergson, nous observons que ce qu'il est convenu d'appeler la pensée occidentale se nourrit des traditions helléniques et judéo-chrétiennes. Et l'histoire de la pensée occidentale est celle de la rencontre de ces deux traditions, avec le lot inhérent d'accords et de désaccords qui régit toute rencontre. Interrogeant la notion de création (dont l'origine est clairement religieuse) dans la philosophie de Bergson, Gouhier, au terme d'une étude précise, montre que ce concept, déjà incorporé à la philosophie cartésienne, fait l'objet d'une appropriation originale dans le bergsonisme'. Notre hypothèse est que la notion de pardon, à l'instar de celle de création, est appelée à occuper l'espace philosophique.

S'il est une notion dont on ne peut guère contester l'origine, c'est bien celle de pardon. Elle est, traditionnellement, clairement circonscrite au domaine religieux et se déploie dans le champ des trois grands monothéismes. La philosophie, quant à elle, est fondée, en première approximation, à lui refuser l'hospitalité dans sa sphère propre, conformément à l'enseignement du rationalisme classique. Cependant, depuis quelques décennies, des philosophes s'efforcent d'acclimater la notion de pardon dans l'espace particulier de la philosophie, mais sans jamais l'abstraire de son lieu religieux de naissance ${ }^{2}$. En effet, la deuxième moitié du XXe siècle, confrontée à la question de l'impardonnable à travers celle de l'imprescriptibilité des crimes contre l'humanité, paraît redonner à la réflexion sur le pardon un intérêt dans la sphère laïque, convoquant ainsi le questionnement philosophique. D'autre part, méditant sur l'irréversibilité du passé et l'imprévisibilité de l'avenir dans les affaires humaines, Hannah Arendt, dans sa construction anthropologique, propose les réponses du pardon et de la promesse. Cette situation singulière où l'on voit une 
notion religieuse investir le champ philosophique, sans renier son terreau originel est assez singulière pour mériter l'attention et l'intérêt de la communauté philosophante.

\section{Le refus philosophique du pardon}

La plupart des grandes philosophies évacuent le pardon de leur territoire, non sans s'appuyer sur de solides arguments. Ainsi les stoïciens ont toujours pris soin de refuser le pardon en avançant deux motifs. D'abord le sage ne doit pas se laisser dominer par ses émotions quelles qu'elles soient car la raison doit toujours l'emporter. Non seulement nous devons ne pas céder à la colère ou à la haine, mais le chagrin, la compassion, la pitié sont autant de concessions à la passion et de reculs de la raison. Cependant, ajoute Épictète, si nous ne pouvons nous empêcher d'éprouver un sentiment, il est préférable que ce soit la pitié plutôt que la haine qui l'emporte. La seconde objection adressée au pardon est son injustice en quelque sorte structurelle. En effet, la remise d'une peine méritée, accordée selon le bon vouloir de l'offensé, du juge ou du prince, vient perturber l'ordre juridique et même le contredire puisque la règle du droit ne serait pas la même pour tous. Cependant l'attitude stoïcienne, par la voix de Sénèque, par exemple, recommande la sévérité mais exclut la cruauté; elle refuse le pardon, mais invite à la clémence («maîtrise de soi lorsqu'on a le pouvoir de punir»); elle réclame la justice mais ne veut point la vengeance; elle applique le droit mais tient compte des circonstances atténuantes; elle punit mais sait remettre partiellement les peines pour permettre un nouveau départ à certains accusés.

Sous des formes analogues, les grandes philosophies rationalistes refusent le pardon. Pour Spinoza, il importe de ne jamais permettre aux méchants de tirer avantage des maux commis en échappant à la justice et, de manière très voisine, Kant assimile le pardon à l'impunité, forme suprême de l'injustice. L'un et l'autre écartent l'esprit de vengeance. Kant, au moment où il condamne «la molle indulgence», en appelle à ce qu'il nomme le pardon mais qui est en réalité seulement le refus de l'implacabilité dans l'application de la peine et non pas l'effacement de la trace de l'offense. Parce que le pardon est contingent, gratuit, il paraît arbitraire et impossible à universaliser. C'est pourquoi le kantisme lui dénie tout fondement. Aussi a-t-on pu soutenir que le pardon «est impossible à qui veut penser, vivre et agir rationnellement, objectivement, avec cohérence et rigueur : le philosophe ne se venge pas, mais ne peut pas pardonner ${ }^{3}$,. 


\section{La reprise philosophique du pardon}

Cette position abrupte est celle d'une certaine philosophie rationaliste, mais n'est pas le dernier mot de la philosophie. En délimitant son territoire formellement distinct de celui de la religion, en déployant sa structure argumentative de manière parfaitement agnostique, la philosophie ne s'interdit pas de puiser son inspiration dans l'expérience religieuse. Par exemple, la notion de personne qui doit à la culture grecque et au droit romain des éléments essentiels de sa généalogie est incontestablement aussi l'héritière de la tradition chrétienne ${ }^{4}$. Ou encore, comme nous l'avons dit en commençant, la notion de création, caractéristique du judaïsme et du christianisme, a fait l'objet d'une reprise originale dans la philosophie de Descartes ou dans celle de Bergson. Enfin la notion de mal radical chez Kant n'estelle pas intégration philosophique de la notion théologique de péché originel? Le pardon, qui est sans justification rationnelle ou juridique, "don gracieux de l'offensé à l'offenseur (Vladimir Jankélévitch)», fait partie de ces notions aptes à prendre place dans le champ philosophique après leur acte de naissance religieux. La philosophie de Hegel réserve au pardon une place singulière dans la dialectique de la "reconnaissance» de l'autre. Le pardon est le moment de la reconnaissance de l'égalité avec autrui, moment où une conscience, dégagée de toute action dans le monde, renonce à son attitude surplombante, arrogante et hypocrite, et cesse de juger ceux qui se compromettent dans l'action.

Mais c'est surtout chez quatre éminents penseurs contemporains que s'est réalisée l'intégration du pardon dans le champ philosophique, Hannah Arendt, Vladimir Jankélévitch, Jacques Derrida et Paul Ricœur. C'est donc en examinant le surgissement de la notion de pardon au sein de ces quatre pensées, assez représentatives de la philosophie contemporaine, que nous pourrons tester la validité de notre hypothèse.

\section{Hannah Arendt : le pardon dans l'action humaine}

Réfléchissant aux conditions de possibilité de l'action humaine, Hannah Arendt indique comment l'homme lutte contre l'imprévisibilité de l'avenir et l'irréversibilité du passé. Par la faculté de promettre, l'homme peut résister à l'indétermination du futur. Par la faculté de pardonner, il peut dénouer les liens du passé. Plus précisément, le passé et son cortège de malheurs ne sauraient être effacés puisque le temps écoulé est par nature impossible à modifier. Mais la mémoire 
du mal (qui est littéralement, en russe, la rancune : zlopamiatsivo, comme le rappelle Jankélévitch) peut être «travaillée». Contre le remords qui emprisonne le coupable dans le passé irréversible, contre la rancune qui enferme la victime dans la même fatalité, surgissent la possibilité du repentir et l'éventualité du pardon qui défont les nœuds du passé. Le mal n'est pas aboli, mais sa mémoire ne paralyse plus ceux qui l'ont subi, ni ceux qui l'ont commis. Un nouvel avenir est alors offert à ceux qui se croyaient victimes à jamais de l'irréversibilité du passé.

Au moment où Hannah Arendt désigne le pardon comme l'une des conditions de l'agir humain, elle en signale l'origine religieuse. "C'est Jésus de Nazareth écrit-elle, qui découvrit le rôle du pardon dans le domaine des affaires humaines. Qu'il ait fait cette découverte dans un contexte religieux, qu'il l'ait exprimée dans un langage religieux, ce n'est pas une raison pour le prendre moins au sérieux en un sens strictement laïc ${ }^{5}$ ".

La tradition chrétienne, en cela héritière de la tradition juive, rappelle que le pardon n'est pas un privilège de Dieu, mais qu'il y a urgence à le corréler avec le pardon humain, ainsi qu'en témoigne la cinquième demande du Notre Père, la prière que Jésus a enseignée à ses disciples. "Remets-nous nos dettes comme nous aussi avons remis à nos débiteurs". Le pardon - qui est exactement la remise de la dette selon l'expérience chrétienne - se voit donc reconnaître un rôle dans les affaires humaines. Le futur peut être à nouveau ouvert, d'autres commencements peuvent être envisagés.

En conclusion de son analyse du pardon et de la promesse, Hannah Arendt souligne le caractère miraculeux de l'action humaine. Et les vues de Jésus, qui a pressenti que la faculté de pardonner libérait les possibilités de l'action, sont comparées à celles de Socrate sur les possibilités de la pensée. "Le miracle qui sauve le monde, le domaine des affaires humaines, de la ruine normale, «naturelle», c'est finalement le fait de la natalité, dans lequel s'enracine la faculté d'agir». C'est parce qu'il y a des hommes nouveaux, parce que, avant d'être un être-pour-la-mort, l'homme est d'abord un être-qui-est-né, qu'une puissante et miraculeuse capacité de renouvellement, est introduite par le fait de la natalité. La Grèce, selon Hannah Arendt, a méconnu cette capacité, que les Évangiles ont annoncée : «Un enfant nous est né6»" 


\section{Vladimir Jankélévitch : grandeur du pardon mais aussi de l'impardonnable}

De son côté, Vladimir Jankélévitch a proposé une méditation philosophique sur le pardon à partir de l'irruption de la Shoah dans l'histoire. Ce grand moraliste a expérimenté dans sa chair de juif et son intelligence de philosophe, l'impossibilité de pardonner ce qui a été, à travers le génocide, une atteinte à l'humanité de l'homme, comme il l'explique dans les textes réunis sous le titre L'imprescriptible?.

Mais à côté de cette position véhémente et pessimiste, Jankélévitch, dans un livre intitulé sobrement Le Pardon', a su trouver des mots d'une grande noblesse pour faire l'éloge du pardon dans sa forme la plus gratuite et la plus désintéressée. Dans cet ouvrage, qu'il tient à nommer "un livre de philosophie» l'auteur a formé le projet de présenter le pardon en utilisant rigoureusement les procédures philosophiques et en opposant à l'éthique stoïcienne, l'éthique juive et chrétienne, lue, il est vrai, à travers le néoplatonisme. Ce n'est point le lieu d'entrer dans le détail de cette éthique que Jankélévitch qualifie d'hyperbolique, ni de disputer de la conformité ou non de son travail avec la théologie. L'essentiel est ici de repérer qu'une notion d'origine religieuse a pu être soumise à une élaboration philosophique. De plus, la comparaison entre stoïcisme et christianisme, considérés comme deux écoles de sagesse, permet de renouer avec une dimension que la philosophie classique a souvent négligée et que Pierre Hadot, par exemple, a récemment réhabilitée en montrant que la philosophie antique avait été aussi un «exercice spirituel».

\section{Jacques Derrida : la «mondialatinisation» du pardon abra- hamique}

Situant sa réflexion sur le pardon dans le sillage de celle de Jankélévitch, Jacques Derrida observe d'abord que le concept d'essence religieuse se dissémine dans des aires culturelles auxquelles il était étranger, comme le Japon et la Corée. Le pardon, qui appartient à un héritage "singulier" (Jacques Derrida l'appelle abrahamique pour rassembler les trois grands monothéismes), est en voie d' "universalisation". C'est ainsi qu'il faut comprendre la prolifération des scènes de repentir et de pardon comme une "mondialatinisation pour prendre en compte l'effet de christianité romaine qui surdétermine aujourd'hui tout le langage du droit, de la 
politique, et même l'interprétation dudit "retour du religieux». Aucun prétendu désenchantement, aucune sécularisation ne vient l'interrompre, bien au contraire ${ }^{9}$ '.

Pour Jacques Derrida, le pardon relève d'une «folie de l'impossible». Avec Jankélévitch, il admet qu'il y a de l'impardonnable, mais ajoute que c'est précisément en ce point qu'un authentique pardon pourrait se manifester. À ses yeux, pour que le pardon soit conforme à son essence de geste purement désintéressé, il faut qu'il revête un degré d'absoluité au point d'être rigoureusement inconditionnel. Si l'on pardonne en vue d'un intérêt plus ou moins masqué, comme il arrive dans les procédures d'amnistie ou de réconciliation, alors on manque la totale gratuité du pardon.

Allant très loin dans la recherche de l'absolu, Jacques Derrida refuse que l'on sépare le coupable de son acte lors du geste de pardon. Car si l'on pardonne à celui qui s'est déjà repenti, pardonne-ton au coupable en tant que tel, ou à un autre désormais meilleur? Si le repentir est la condition du pardon, celui-ci ne mérite plus son nom car il est, par nature selon notre philosophe, rigoureusement inconditionnel. On comprend que le pardon doive être chose rare, exceptionnelle, au point d'être fou, inintelligible, et, surtout a-juridique. Empruntant à Jankélévitch l'idée d'une "éthique hyperbolique», Jacques Derrida donne le sentiment d'avoir donné une coloration "gnostique», selon le mot de Paul Ricœur, à la notion de pardon qui se trahirait dès lors qu'il rencontrerait un début de réalisation. On retrouverait aisément le même schéma dans l'ensemble de la philosophie derridienne en explorant d'autres concepts qui ont une consistance propre dans la sphère de l'héritage abrahamique, tels que l'hospitalité, la justice ou le don.

\section{Paul Ricœur : le pardon difficile}

Reconnaissant sa dette à l'égard des trois philosophes que nous avons mentionnés (Jankélévitch, Arendt, Derrida), Paul Ricœur, au terme d'un ouvrage remarquable, La Mémoire, l'histoire, l'oubli, a consacré un épilogue substantiel à la question du pardon. Le pardon n'est pas impossible mais difficile, préfère dire Ricœur. Peut-on déceler dans cette thèse philosophique une influence des convictions chrétiennes de l'auteur? Probablement, mais à la condition de ne pas confondre les conditions profondes et intimes de l'élaboration d'une pensée et sa dynamique singulière et autonome. II faut distinguer, d'une façon générale, "le cours souterrain de la motivation et le 
développement maîtrisé de l'argumentation ${ }^{10}$ ". La philosophie de Ricœur n'est pas une philosophie chrétienne. Si elle croise les cheminements du religieux, c'est au sens le plus vaste de ce mot, comme on le voit, par exemple, à travers la réflexion fine sur le pardon et l'oubli dans Athènes, qui clôt l'ouvrage.

L'amnistie dans la cité athénienne fonctionne comme une institution de l'oubli, mais fondée sur une dénégation de la violence fondatrice sur laquelle se construit la paix civique. La mémoire oublieuse est la condition du politique. "Tel est l'enjeu spirituel de l'amnistie : faire taire le non-oubli de la mémoire. C'est pourquoi le politique grec a besoin du religieux pour soutenir la volonté d'oubli de l'inoubliable, sous la forme d'imprécations à l'horizon du parjure ${ }^{11 \text { ". }}$

On le sait, le religieux grec, n'occupe pas le même espace que le religieux juif ou encore le religieux chrétien, mais c'est aussi ce dernier qui est mobilisé à la fin de la réflexion de Ricœur après qu'il fût entré auparavant en discussion avec Arendt et Derrida, eux-mêmes tributaires de la représentation qu'ils se sont faite du pardon dans les religions abrahamiques ${ }^{12}$. Et c'est enfin une philosophie peu suspecte de confusion entre le religieux et le philosophique, le kantisme, et plus précisément La Religion dans les limites de la simple raison, que Ricœur mobilise pour arriver à sa conclusion. II recourt à la philosophie kantienne de la religion pour rappeler que la disposition au bien est originaire, et que nous avons à viser la restauration en nous de cette disposition primitive au bien.

Sans doute, la thématique kantienne n'est pas adaptée à la problématique du pardon et de la repentance, mais, malgré les critiques adressées aux formes historiques des religions monothéistes par Kant, la conviction d'une possible «libération du fond de la bonté de l'homme" s'appuie sur "une relecture philosophique des vieux mythes traitant de l'origine méta - ou transhistorique du mal».

(...) Seraient alors à verser au service de cet immense projet de restauration, d'une part, les symboles qui - tels celui du serviteur souffrant et de son expression christique - nourrissent l'imaginaire juif et chrétien; d'autre part, les institutions métapolitiques - telles que, en chrétienté, les formes visibles de l'Église placées à l'égard de ce dépôt imaginaire dans la double position de disciple et de gardien. C'est à ces symboles et à ces institutions qu'est consacrée la suite de La Religion..., 
que Kant déploie, il est vrai, sur un ton de plus en plus en plus véhément à l'adresse des formes historiques revêtues par ce religieux de base que nous dirions aujourd'hui être celui des religions du Livre.

C'est sur l'arrière-plan de cette lecture philosophique du religieux occidental que se détache l'énigme du pardon dans l'espace de sens de ces religions ${ }^{13}$.

De ce quadruple parcours, il ressort nettement que la notion de pardon est en train de trouver droit de cité dans l'espace philosophique, ce qui ne l'empêche pas de poursuivre sa carrière dans l'espace religieux où sa structure et ses exigences sont assurément de nature différente. Mais notre brève enquête, à partir d'un concept d'origine religieuse fait apparaître que nous pouvons philosopher d'une manière complètement indépendante du mode de penser religieux sans pour autant nier la réserve de pensée contenue dans l'expérience religieuse.

\section{Jacques Ricot jacques.ricot@wanadoo.fr}

1. Henri Gouhier, Bergson dans l'histoire de la pensée occidentale, Paris, Vrin, 1989, $134 \mathrm{p}$.

2. J'ai tenté modestement de contribuer à cette acclimatation : Jacques RICOT, Peuton tout pardonner?, Nantes, Pleins Feux, $2^{\theta}$ édition, 2001, 96 p.

3. Bernard Rousset, “La possibilité philosophique du pardon. Spinoza, Kant, Hegel» dans Le Pardon. Le Point théologique, Beauchesne, vol. 45, p. 196.

4. Philippe Cormier, Généalogie de personne, Paris, Critérion, 1994.

5. Hannah Arendt, La condition de l'homme moderne, Paris, Presse-Pocket, 1992, p. 304.

6. Ibid., p. 314. En réalité, la phrase est prononcée par le prophète Isaïe.

7. Vladimir Jankélévitch, L'Imprescriptible, Paris, Seuil, 1986.

8. Le Pardon, Paris, Aubier-Montaigne, 1967.

9. Jacques Derrida, Foi et Savoir suivi de Le siècle et le pardon, Paris, Seuil, 2001, p. 108.

10. Paul Ricœur, La Mémoire, l'histoire, l'oubli, Paris, Seuil, 2000, p. 643.

11. Ibid., p. 651.

12. Ibid., p. 633 et $638-639$.

13. Ibid., p. 640-641. 